EUROPEAN JOURNAL OF PURE AND APPLIED MATHEMATICS

Vol. 12, No. 2, 2019, 418-431

ISSN 1307-5543 - www.ejpam.com

Published by New York Business Global

\title{
Convergence of $\beta$-Modified Jacobi-Perron algorithm over the field of formal power series
}

\author{
Amara Chandoul ${ }^{1}$, Fahad Aljuaydi ${ }^{2, *}$ \\ 1 Departamento de Matemática, Universidade de Brasília, Campus Universitário Darcy \\ Ribeiro Brasilia - DF 70910-900, Brazil \\ 2 Department of Mathematics, College of Sciences and Humanities, \\ Prince Sattam bin Abdulaziz University, Al-Kharj, Saudi Arabia
}

\begin{abstract}
The aim of this paper is to study multidimentional $\beta$-continued fraction algorithm over the field of formal power series. In the case of the Modified Jacobi-Perron algorithm, we prove that it converges.
\end{abstract}

2010 Mathematics Subject Classifications: 40A15

Key Words and Phrases: $\beta$-Continued fractions, Modidied Jacobi-Perron algorithm, convergence, formal power series.

\section{Introduction}

In [4], we studied multidimensional continued fraction algorithm over the field of formal power series. In the case of the Brun algorithm by using its homogenous version, we prove that it converges. In this paper, we study multidimentional $\beta$-continued fraction in the case of the Modified Jacobi Perron algorithm (MJPA), we prove that it converges.

\section{The field of formal power series}

In order to state our results, we need to introduce some basic notion of the field of formal power series. Let $\mathbb{F}_{q}$ be a field with $q$ elements of characteristic $p, \mathbb{F}_{q}[X]$ the set of polynomials of coefficients in $\mathbb{F}_{q}$ and $\mathbb{F}_{q}(X)$ its field of fractions. The set $\mathbb{F}_{q}\left(\left(X^{-1}\right)\right)$ is the field of formal power series over $\mathbb{F}_{q}$

$$
\mathbb{F}_{q}\left(\left(X^{-1}\right)\right)=\left\{f=\sum_{j=s}^{+\infty} f_{j} X^{-j}: f_{j} \in \mathbb{F}_{q}, s \in \mathbf{Z}\right\} .
$$

*Corresponding author.

DOI: https://doi.org/10.29020/nybg.ejpam.v12i2.3391

Email addresses: amarachandoul@yahoo.fr (A. Chandoul), f.m.427@hotmail.com (F. Aljuaydi) 
Let $f=\sum_{j=s}^{+\infty} f_{j} X^{-j} \in \mathbb{F}_{q}\left(\left(X^{-1}\right)\right)$, where $f_{s} \neq 0$. We denote its polynomial part by $[f]$ and by $\{f\}$ its fractional part. We remark that $f=[f]+\{f\}$. We define a non-archimedean absolute value on $\mathbb{F}_{q}\left(\left(X^{-1}\right)\right)$ by $|f|=e^{-s}$ and $|0|=0$. It is clear that, for any $P \in \mathbb{F}_{q}[X]$, $|P|=e^{\operatorname{deg} P}$ and, for any $Q \in \mathbb{F}_{q}[X]$, such that $Q \neq 0,\left|\frac{P}{Q}\right|=e^{\operatorname{deg} P-\operatorname{deg} Q}$.

Let $\beta_{0} \in \mathbb{F}_{q}\left(\left(X^{-1}\right)\right) \backslash\{0\}$, then, we define

$$
\mathbb{L}=\left\{\omega \in \mathbb{F}_{q}\left(\left(X^{-1}\right)\right),|\varphi|<\left|\beta_{0}\right|\right\},
$$

which is a compact abelian group with the addition and the metric $d(\varphi, \omega)=|\varphi-\omega|$ : $\forall \varphi, \omega \in \mathbb{F}_{q}\left(\left(X^{-1}\right)\right)$.

Now, for $1 \leq j \leq n$, we put

$$
\mathbb{L}_{j}^{(n)}=\left\{\left(\varphi_{1}, \cdots, \varphi_{n}\right) \in \mathbb{L}^{n},\left\{\begin{array}{l}
\left|\varphi_{j}\right|>\left|\varphi_{i}\right| \text { for } 1 \leq i<j, \\
\left|\varphi_{j}\right| \geq\left|\varphi_{i}\right| \text { for } j<i \leq n
\end{array}\right\},\right.
$$

and

$$
\mathbb{L}_{j}^{n}=\mathbb{L} \times \mathbb{L} \times \cdots \times \mathbb{L}, n \text { times }
$$

then

$$
\mathbb{L}_{j}^{(n)} \subset \mathbb{L}_{j}^{n} \text { and } \mathbb{L}^{n}=\bigcup_{1 \leq i \leq n} \mathbb{L}_{i}^{(n)}
$$

\section{3. $\beta$-Continued fraction in $\mathbb{F}_{q}\left(\left(X^{-1}\right)\right)$}

Let $\beta=\left(\beta_{i}\right)_{i \in \mathbb{Z}}$ with $\beta_{i} \in \mathbb{F}_{q}\left(\left(X^{-1}\right)\right) \backslash\{0\}$, such that $\operatorname{deg}\left(\beta_{i}\right)_{i \in \mathbb{Z}}$ is a strictly increasing sequence of integers. $\beta$ is called base sequence. Let

$$
\mathcal{S}=\left\{\left(d_{i}\right)_{-\infty<i \leq k}: k \in \mathbb{Z}, d_{i} \in \mathbb{F}_{q}[X], \operatorname{deg} d_{i}<\operatorname{deg} \beta_{i+1}-\operatorname{deg} \beta_{i}\right\}
$$

be the set of admissible digit strings associated to the sequence $\beta$.

Lemma 1. Let $\beta=\left(\beta_{i}\right)_{i \in \mathbb{Z}}$ be a base sequence and $\mathcal{S}$ the associated set of admissible digit strings. Then each $\omega \in \mathbb{F}_{q}\left(\left(X^{-1}\right)\right)$ admits a unique representation of the form

$$
\omega=\sum_{-\infty<i \leq k} d_{i} \beta_{i}, \quad\left(d_{i}\right)_{-\infty<i \leq k} \in \mathcal{S}
$$

The above lemma justifies that we call $(\beta, \mathcal{S})$ a digit system. Conversly, a formal power series associated to a given string in the digit system $(\beta, \mathcal{S})$ is given by the evaluation map

$$
\pi: \mathcal{S} \longrightarrow \mathbb{F}_{q}\left(\left(X^{-1}\right)\right),\left(d_{i}\right)_{-\infty<i \leq k} \longrightarrow \sum_{-\infty<i \leq k} d_{i} \beta_{i} .
$$

If a representation ends in infinitely many zeros, it said to be finite, and the final zeros are omitted. 
If all the $s_{i}$ on the right hand side of the radix point are zeros, the representation is said to be an integer representation.

The set of all $\omega \in \mathbb{F}_{q}\left(\left(X^{-1}\right)\right)$ admitting an integer representation is called the set of $\beta$-integers. For $\omega \in \mathbb{F}_{q}\left(\left(X^{-1}\right)\right)$, we define the $\beta$-integer and the $\beta$-fractional part by

$$
[\beta]_{\beta}=\pi\left(d_{k} \cdots d_{0}\right)_{\beta} \text { and }\{\beta\}_{\beta}=\pi\left(d_{-1} d_{-2} \cdots\right)_{\beta},
$$

respectively.

Now we are in a position to introduce our new algorithm, called $\beta$-continued fraction algorithm. The study of this algorithm is similar to the study of the usual continued fraction expansions. Let $\beta=\left(\beta_{i}\right)_{i \in \mathbb{Z}}$ be a base sequence and let $\mathcal{H}^{\prime}{ }_{0}(\beta)=\left\{d \beta_{0} \in \mathbb{F}_{q}[X], 0<\operatorname{deg} d<\operatorname{deg} \beta_{1}-\operatorname{deg} \beta_{0}\right\}$, $\mathcal{H}^{\prime \prime}{ }_{0}(\beta)=\left\{d \beta_{0} \in \mathbb{F}_{q}[X], \operatorname{deg} d<\operatorname{deg} \beta_{1}-\operatorname{deg} \beta_{0}\right\}$,

$\mathcal{H}_{n}(\beta)=\left\{d_{0} \beta_{0}+\cdots+d_{n} \beta_{n}, d_{i} \in \mathbb{F}_{q}[X], \operatorname{deg} d_{i}<\operatorname{deg} \beta_{i+1}-\operatorname{deg} \beta_{i}, d_{n} \neq 0\right\}$ for all $n \geq 1$, and

$$
\begin{aligned}
& \mathcal{H}(\beta)=\mathcal{H}^{\prime}{ }_{0}(\beta) \cup \bigcup_{n \geq 1} \mathcal{H}_{n}(\beta), \\
& \mathcal{I}(\beta)=\mathcal{H}^{\prime \prime}{ }_{0}(\beta) \cup \bigcup_{n \geq 0} \mathcal{H}_{n}(\beta) .
\end{aligned}
$$

Remark 1. Note that $|z| \geq\left|\beta_{0}\right|$ for all $z \in \mathcal{I}(\beta)$ and $|z|>\left|\beta_{0}\right|$ for all $z \in \mathcal{H}(\beta)$.

We can define the $\beta$-continued fraction by the $\beta$-transformation $T_{\beta}$ on $D\left(0,\left|\beta_{0}\right|\right)$, which is given by the following mapping

$$
\begin{aligned}
& \mathbf{T}_{\beta}: D\left(0,\left|\beta_{0}\right|\right) \rightarrow \quad D\left(0,\left|\beta_{0}\right|\right) \\
& \omega \mapsto\left\{\begin{array}{cl}
\left\{\frac{\beta_{0}^{2}}{\omega}\right\}_{\beta} & \text { if } f \neq 0 \\
0 & \text { else }
\end{array}\right.
\end{aligned}
$$

For any base sequence $\beta$, the so-called $\beta$-continued fraction is introduced in [7]. A $\beta$-continued fraction is an expression of the form

$$
\omega=a_{0}+\frac{\beta_{0}^{2}}{a_{1}+\frac{\beta_{0}^{2}}{\cdots+\frac{\beta_{0}^{2}}{a_{n}+\cdots}}}=\left[a_{0} ; a_{1}, \cdots\right]_{\beta},
$$

where $a_{0} \in \mathcal{I}(\beta)$ and $a_{i} \in \mathcal{H}(\beta)$ for $i \geq 1$. It is easy to prove that $\operatorname{deg} a_{i}>\operatorname{deg} \beta_{0}$ for all $i \geq 1$.

Remark 2. If $\beta=\left(X^{i}\right)_{i \in \mathbb{N}}$, then the transformation $T_{\beta}$ describe the regular continued fraction over the field of formal power series and has been introduced by Artin [8]. 


\section{Multidimensional continued fractions}

Let $B \subset E$ and $T: B \longrightarrow B$ be a map. The pair $(B, T)$ is called a fibred system if there exists a finite or countable partition $\{B(P): P \in I\}$ of $B$, where $I \subset \mathbb{F}_{q}[X]^{n}$, such that the restriction of $T$ to any $B(P)$ is an injective map. As $E$ is a normed space, we assume that a system defines an algorithm of multidimensional continued fractions if for all $P=\left(P_{1}, \ldots, P_{n}\right) \in I$, there exists an $(n+1) \times(n+1)$ invertible matrix $\alpha(P)=\left(C_{i, j}\right)$ with entries in $\mathbb{F}_{q}[X]$ such that if $y=T f$ where $f \in B(P)$, then $y_{i}=\frac{C_{i 0}+\sum_{j=1}^{n} C_{i j} f_{j}}{C_{00}+\sum_{j=1}^{n} C_{0 j} f_{j}}$ for all $1 \leq i \leq n$.

The map $T$ is called a multidimensional continued fraction algorithm. For all $1 \leq$ $i \leq n$, if $f \in B\left(P^{(1)}\right)$, then $T^{i} f \in B\left(P^{(i)}\right)$. The sequence $P^{(1)}, P^{(2)}, \ldots, P^{(n)}, \ldots$ is called the expansion of $f$ by the algorithm $T$.

Let $\beta(P)=\left(B_{i, j}\right)$ be the inverse matrix of $\alpha(P)$, we set

$$
\begin{aligned}
\beta\left(P^{(1)}, \cdots, P^{(s)}\right) & =\beta\left(P^{(1)}\right) \cdots \beta\left(P^{(s)}\right) \\
& =\left(\left(B_{i j}^{(s)}\right)\right),
\end{aligned}
$$

where $0 \leq i, j \leq n$, then $y=T^{s} f$ if, and only if,

$$
f_{i}=\frac{B_{i 0}^{(s)}+\sum_{g=1}^{n} B_{i g}^{(s)} y_{g}}{B_{00}^{(s)}+\sum_{g=1}^{n} B_{0 g}^{(s)} y_{g}} .
$$

The algorithm $T$ is said convergent, if for all $f \in B$,

$$
\lim _{s \rightarrow+\infty}\left(\frac{B_{10}^{(s)}}{B_{00}^{(s)}}, \ldots, \frac{B_{n 0}^{(s)}}{B_{00}^{(s)}}\right)=f .
$$

The vectors $\left(\frac{B_{10}^{(s)}}{B_{00}^{(s)}}, \ldots, \frac{B_{n 0}^{(s)}}{B_{00}^{(s)}}\right)$ are the convergents of $f$.

\section{Definitions}

In this section, we define a map $T_{\beta}$ which is arisen from $\beta$-MJPA.

Let $\beta=\left(\beta_{i}\right)_{i \in \mathbb{Z}}$ be a base sequense. The map $T_{\beta}: \mathbb{L}^{n} \rightarrow \mathbb{L}^{n}$ by

$$
\begin{aligned}
T_{\beta}\left(\varphi_{1}, \ldots, \varphi_{n}\right) & =\left(\frac{\varphi_{2}}{\varphi_{j}}, \ldots, \frac{\varphi_{j-1}}{\varphi_{j}},\left\{\frac{\beta_{0}^{2}}{\varphi_{j}}\right\}_{\beta},\left\{\frac{\varphi_{j+1}}{\varphi_{j}}\right\}_{\beta}, \ldots,\left\{\frac{\varphi_{n}}{\varphi_{j}}\right\}_{\beta}\right) \\
& =\left(\frac{\varphi_{2}}{\varphi_{j}}, \ldots, \frac{\varphi_{j-1}}{\varphi_{j}}, \frac{1}{\varphi_{j}}-a_{n+1}, \frac{\varphi_{j+1}}{\varphi_{j}}-a_{j+1}, \ldots, \frac{\varphi_{n}}{\varphi_{j}}-a_{n}\right)
\end{aligned}
$$



where $a_{n+1}\left[\frac{\beta_{0}^{2}}{\varphi_{j}}\right]_{\beta}$ and $a_{i}=\left[\frac{\varphi_{i}}{\varphi_{j}}\right]_{\beta}: i \geq j+1$, for $\left(\varphi_{1}, \ldots, \varphi_{n}\right) \in \mathbb{L}_{j}^{n},\left(\varphi_{1}, \ldots, \varphi_{n}\right) \neq$ $(0, \ldots, 0)$ and $T_{\beta}(0, \ldots, 0)=(0, \ldots, 0)$.

For $s \geq 1$, we put $\left(\varphi_{1}^{(s)}, \ldots, \varphi_{n}^{(s)}\right)=T_{\beta}^{s}\left(\varphi_{1}, \ldots, \varphi_{n}\right)$ and $a_{i}^{(s)}=a_{i}\left(\varphi_{1}^{(s-1)}, \ldots, \varphi_{n}^{(s-1)}\right)=$ $\left(0, \cdots, 0,\left[\frac{\beta_{0}^{2}}{\varphi_{j}^{(s-1)}}\right]_{\beta},\left[\frac{\varphi_{j+1}^{(s-1)}}{\varphi_{j}^{(s-1)}}\right]_{\beta}, \ldots,\left[\frac{\varphi_{n}^{(s-1)}}{\varphi_{j}^{(s-1)}}\right]_{\beta}\right)=\left(0, \cdots, 0, a_{n+1}, a_{j+1}, \cdots, a_{n}\right)$ for $1 \leq$ $i \leq n+1$, that is

$$
\begin{aligned}
T_{\beta}^{s}\left(\varphi_{1}, \ldots, \varphi_{n}\right) & =T\left(\varphi_{1}^{(s-1)}, \ldots, \varphi_{n}^{(s-1)}\right) \\
& =\left(\frac{\varphi_{2}^{(s-1)}}{\varphi_{j}^{(s-1)}}, \ldots, \frac{\varphi_{j-1}^{(s-1)}}{\varphi_{j}^{(s-1)}},\left\{\frac{\beta_{0}^{2}}{\varphi_{j}^{(s-1)}}\right\}_{\beta},\left\{\frac{\varphi_{j+1}^{(s-1)}}{\varphi_{j}^{(s-1)}}\right\}_{\beta}, \ldots,\left\{\frac{\varphi_{n}^{(s-1)}}{\varphi_{j}^{(s-1)}}\right\}_{\beta}\right) \\
& =\left(\frac{\varphi_{2}^{(s-1)}}{\varphi_{j}^{(s-1)}}, \ldots, \frac{\varphi_{j-1}^{(s-1)}}{\varphi_{j}^{(s-1)}}, \frac{1}{\varphi_{j}^{(s-1)}}-a_{n+1}^{(s)}, \frac{\varphi_{j+1}^{(s-1)}}{\varphi_{j}^{(s-1)}}-a_{j+1}^{(s)}, \ldots, \frac{\varphi_{n}^{(s-1)}}{\varphi_{j}^{(s-1)}}-a_{n}^{(s)}\right)
\end{aligned}
$$

for $\left(\varphi_{1}^{(s-1)}, \ldots, \varphi_{n}^{(s-1)}\right) \in \mathbb{L}_{j}^{n}$. Also we put $\kappa(s):=j$ such that

$$
\operatorname{deg} \varphi_{j}^{(s-1)}>\varphi_{i}^{(s-1)} \text { for } 1 \leq i<j \text { and } \operatorname{deg} \varphi_{j}^{(s-1)} \geq \varphi_{i}^{(s-1)} \text { for } j<i \leq n .
$$

\subsection{The matrix}

Let $\left(\varphi_{1}, \ldots, \varphi_{n}\right) \in \mathbb{L}_{j}^{n},\left(\varphi_{1}, \ldots, \varphi_{n}\right) \neq(0, \ldots, 0)$. We define the $(n+1) \times(n+1)$ matrix $M=\left(m_{i_{1} i_{2}}\right), m_{i_{1} i_{2}} \in \mathbb{F}_{q}\left(\left(X^{-1}\right)\right)$, associated to $\left(\varphi_{1}, \ldots, \varphi_{n}\right)$ in the following way :

(i) $1 \leq i_{2} \leq n, i_{2} \neq j$

$$
m_{i_{1} i_{2}}=\delta_{i_{1} i_{2}} \text { for } 1 \leq i_{1} \leq n+1 \text {, }
$$

(ii) $i_{2}=j$

$$
m_{i_{1} i_{2}}=\left\{\begin{array}{l}
1 \text { for } i_{1}=n+1 \\
0 \text { for } 1 \leq i_{1} \leq n
\end{array}\right.
$$

(iii) $i_{2}=n+1,1 \leq i_{1} \leq n+1$

$$
m_{i_{1} i_{2}}=a_{i_{1}}
$$


that is,

$$
M=M\left(\varphi_{1}, \ldots, \varphi_{n}\right)=\left(\begin{array}{ccccccccc}
1 & 0 & \ldots & 0 & 0 & 0 & \ldots & 0 & 0 \\
0 & 1 & \ldots & 0 & 0 & 0 & \ldots & 0 & 0 \\
\vdots & \vdots & \ddots & \vdots & \vdots & \vdots & \ddots & \vdots & \vdots \\
0 & 0 & \ldots & 1 & 0 & 0 & \ldots & 0 & 0 \\
0 & 0 & \ldots & 0 & 0 & 0 & \ldots & 0 & 1 \\
0 & 0 & \ldots & 0 & 0 & 1 & \ldots & 0 & a_{j+1} \\
\vdots & \vdots & \ddots & \vdots & \vdots & \vdots & \ddots & \vdots & \vdots \\
0 & 0 & \ldots & 0 & 0 & 0 & \ldots & 1 & a_{n} \\
0 & 0 & \ldots & 0 & 1 & 0 & \ldots & 0 & a_{n+1}
\end{array}\right)
$$

For $\left(\varphi_{1}, \ldots, \varphi_{n}\right)=(0, \ldots, 0)$, we define $M$ the $(n+1) \times(n+1)$ unit matrix $I_{n+1}$. We put

$$
\begin{gathered}
M^{(0)}=I_{n+1}, \\
M^{(s)}=M\left(\varphi_{1}^{(s-1)}, \ldots, \varphi_{n}^{(s-1)}\right) \text { for } s \geq 1,
\end{gathered}
$$

where $\left(\varphi_{1}^{(0)}, \ldots, \varphi_{n}^{(0)}\right)=\left(\varphi_{1}, \ldots, \varphi_{n}\right)$. Since, we consider the columns of the matrix $M^{(1)}, \ldots, M^{(s)}$, we denote

$$
M^{(1)} \ldots M^{(s)}=\left(\begin{array}{ccccc}
A_{11}^{(s)} & \ldots & \ldots & A_{1 n}^{(s)} & B_{1}^{(s)} \\
\vdots & \vdots & & & \vdots \\
A_{\kappa(s) 1}^{(s)} & \ldots & \ldots & A_{\kappa(s) n}^{(s)} & B_{j}^{(s)} \\
\vdots & \vdots & & & \vdots \\
A_{n 1}^{(s)} & \ldots & \ldots & A_{n n}^{(s)} & B_{n}^{(s)} \\
A_{01}^{(s)} & \ldots & \ldots & A_{0 n}^{(s)} & B_{0}^{(s)}
\end{array}\right)
$$

and

$$
M^{(0)}=\left(\begin{array}{cccc}
B_{1}^{(-d)} & \ldots & B_{1}^{(-1)} & B_{1}^{(0)} \\
\vdots & \vdots & & \vdots \\
B_{n}^{(-n)} & \ldots & A_{n n}^{(s)} & B_{n}^{(0)} \\
A_{0}^{(-n)} & \ldots & B_{0}^{(-1)} & B_{0}^{(0)}
\end{array}\right)
$$

Using definition of $B_{0}^{(s)}$, it is clear that $\operatorname{deg} B_{0}^{(s)}=\sum_{i=1}^{s} \operatorname{deg} a_{n+1}^{(i)}$ which we use often. $B_{0}^{(s)}$ will be the denominator of the $s$-th convergent and $B_{i}^{(s)}, 1 \leq i \leq n$, will be numerator. 
Evidently,

$$
\begin{aligned}
& M^{(1)} \ldots M^{(s)} \\
& =\left(\begin{array}{ccccc}
A_{11}^{(s-1)} & \ldots & \ldots & A_{1 n}^{(s-1)} & B_{1}^{(s-1)} \\
\vdots & \vdots & & & \vdots \\
A_{\kappa(s) 1}^{(s-1)} & \ldots & \ldots & A_{\kappa(s) n}^{(s-1)} & B_{\kappa(s)}^{(s-1)} \\
\vdots & \vdots & & & \vdots \\
A_{n 1}^{(s-1)} & \ldots & \ldots & A_{n n}^{(s-1)} & B_{n}^{(s-1)} \\
A_{01}^{(s-1)} & \ldots & \ldots & A_{0 n}^{(s-1)} & B_{0}^{(s-1)}
\end{array}\right)\left(\begin{array}{ccccccccc}
1 & 0 & \ldots & 0 & 0 & 0 & \ldots & 0 & 0 \\
0 & 1 & \ldots & 0 & 0 & 0 & \ldots & 0 & 0 \\
\vdots & \vdots & \ddots & \vdots & \vdots & \vdots & \ddots & \vdots & \vdots \\
0 & 0 & \ldots & 1 & 0 & 0 & \ldots & 0 & 0 \\
0 & 0 & \ldots & 0 & 0 & 0 & \ldots & 0 & 1 \\
0 & 0 & \ldots & 0 & 0 & 1 & \ldots & 0 & a_{j+1} \\
\vdots & \vdots & \ddots & \vdots & \vdots & \vdots & \ddots & \vdots & \vdots \\
0 & 0 & \ldots & 0 & 0 & 0 & \ldots & 1 & a_{n} \\
0 & 0 & \ldots & 0 & 1 & 0 & \ldots & 0 & a_{n+1}
\end{array}\right) \\
& =\left(\begin{array}{cccccccc}
A_{11}^{(s-1)} & \ldots & A_{1, \kappa(s)-1}^{(s-1)} & B_{1}^{(s-1)} & A_{1, \kappa(s)+1}^{(s-1)} & \ldots & A_{1 n}^{(s-1)} & B_{1}^{(s)} \\
\vdots & \vdots & \vdots & \vdots & \vdots & \vdots & \vdots & \vdots \\
A_{\kappa(s) 1}^{(s-1)} & \ldots & A_{\kappa(s), \kappa(s)-1}^{(s-1)} & B_{\kappa(s)}^{(s-1)} & A_{\kappa(s), \kappa(s)+1}^{(s-1)} & \ldots & A_{\kappa(s), n}^{(s-1)} & B_{\kappa(s)}^{(s)} \\
\vdots & \vdots & \vdots & \vdots & \vdots & \vdots & \vdots & \vdots \\
A_{n 1}^{(s-1)} & \ldots & A_{n, 1)-1}^{(s-1)} & B_{n}^{(s-1)} & A_{n, \kappa(s)+1}^{(s-1)} & \ldots & A_{n n}^{(s-1)} & B_{n}^{(s)} \\
A_{01}^{(s-1)} & \ldots & A_{0, \kappa(s)-1}^{(s-1)} & B_{0}^{(s-1)} & A_{0, \kappa(s)+1}^{(s-1)} & \ldots & A_{0 n}^{(s-1)} & B_{0}^{(s)}
\end{array}\right) .
\end{aligned}
$$

where $B_{i}^{(s)}=A_{i \kappa(s)}^{(s-1)}+\sum_{k=\kappa(s)+1}^{n} a_{k}^{(s)} A_{i k}^{(s-1)}+a_{n+1}^{s} B_{i}^{s-1}, 0 \leq i \leq n$.

Since $\operatorname{det} M^{(1)} \ldots M^{(s)}= \pm 1$, which follows from (4), we see that $B_{0}^{(s)}, \ldots, B_{n-1}^{(s)}$ and $B_{n}^{(s)}$ have no non-trivial common factor. By a simple calculation for $\left(\varphi_{1}, \ldots, \varphi_{n}\right) \in \mathbb{L}_{\kappa(s)}^{n}$ , we see that

(i) $i_{2} \neq \kappa(s), n+1$

$$
A_{i_{1} i_{2}}^{(s)}=A_{i_{1} i_{2}}^{(s-1)} \text { for } 1 \leq i_{1} \leq n,
$$

(ii) $i_{2}=\kappa(s)$

$$
A_{i_{1} i_{2}}^{(s)}=B_{i_{1}}^{(s-1)} \text { for } 0 \leq i_{1} \leq n,
$$

(iii) $i_{2}=n+1$

$$
A_{i_{1} i_{2}}^{(s)}=B_{i_{1}}^{(s)}=B_{i}^{(s)}=A_{i \kappa(s)}^{(s-1)}+\sum_{k=\kappa(s)+1}^{n} a_{k}^{(s)} A_{i k}^{(s-1)}+a_{n+1}^{s} B_{i}^{s-1} \text { for } 0 \leq i \leq n .
$$

From (5), we find that $B_{i}^{(s)}$ increases as $s$ increases and

$$
\operatorname{deg} B_{i_{1}}^{(s)}>\operatorname{deg} A_{i_{1}, \kappa(s)}^{(s)}>\operatorname{deg} A_{i_{1}, i_{2}}^{(s)}
$$


if $i_{2} \neq \kappa(s), n+1$ for $0 \leq i_{1} \leq n$. We put

$$
M^{(1)} \ldots M^{(s)}\left(\begin{array}{c}
\varphi_{1}^{(s)} \\
\vdots \\
\varphi_{n}^{(s)} \\
1
\end{array}\right)=\left(\begin{array}{c}
A_{11}^{(s)} \varphi_{1}^{(s)}+\ldots+A_{1 n}^{(s)} \varphi_{n}^{(s)}+B_{1}^{(s)} \\
\vdots \\
A_{n 1}^{(s)} \varphi_{1}^{(s)}+\ldots+A_{n n}^{(s)} \varphi_{n}^{(s)}+B_{n}^{(s)} \\
A_{01}^{(s)} \varphi_{1}^{(s)}+\ldots+A_{0 n}^{(s)} \varphi_{n}^{(s)}+B_{0}^{(s)}
\end{array}\right)
$$

and obtain following theorem.

Theorem 1. For any $\left(\varphi_{1}, \ldots, \varphi_{n}\right) \in \mathbb{L}^{n}$, we have

$$
\varphi_{i}=\frac{A_{i 1}^{(s)} \varphi_{1}^{(s)}+\ldots+A_{i n}^{(s)} \varphi_{n}^{(s)}+B_{i}^{(s)}}{A_{01}^{(s)} \varphi_{1}^{(s)}+\ldots+A_{0 n}^{(s)} \varphi_{n}^{(s)}+B_{0}^{(s)}}, \text { for } 1 \leq i \leq n,
$$

whenever $T_{\beta}^{s^{\prime}}\left(\varphi_{1}, \ldots, \varphi_{n}\right) \neq(0, \ldots, 0)$, for any $0 \leq s^{\prime} \leq s$.

Proof. We prove the theorem using the method of mathematical induction. For $n=1$, we have from the definition, for $\left(\varphi_{1}, \ldots, \varphi_{n}\right) \in \mathbb{L}_{j}^{(n)}$,

$$
\begin{aligned}
T_{\beta}\left(\varphi_{1}, \ldots, \varphi_{n}\right) & =\left(\varphi_{1}^{(1)}, \ldots, \varphi_{n}^{(1)}\right) \\
& =\left(\frac{\varphi_{2}}{\varphi_{j}}, \ldots, \frac{\varphi_{j-1}}{\varphi_{j}}, \frac{1}{\varphi_{j}}-a_{n+1}^{(1)}, \frac{\varphi_{j+1}}{\varphi_{j}}-a_{j+1}^{(1)}, \ldots, \frac{\varphi_{n}}{\varphi_{j}}-a_{n}^{(1)}\right)
\end{aligned}
$$

Then

$$
\varphi_{i}=\left\{\begin{array}{llc}
\frac{1 . \varphi_{i}^{(1)}}{1 . \varphi_{j}^{(1)}+a_{n+1}^{(1)}} & \text { for } & 1 \leq i<j \\
\frac{1}{1 . \varphi_{j}^{(1)}+a_{n+1}^{(1)}} & \text { for } & i=j \\
\frac{1 . \varphi_{i}^{(1)}+a_{i}^{(1)}}{1 . \varphi_{j}^{(1)}+a_{n+1}^{(1)}} & \text { for } & j<i \leq n
\end{array}\right.
$$

On the other hand, for $\left(\varphi_{1}, \ldots, \varphi_{n}\right) \in \mathbb{L}_{j}^{(n)}$,

$$
\frac{A_{i 1}^{(1)} \varphi_{1}^{(1)}+\ldots+A_{i n}^{(1)} \varphi_{n}^{(1)}+B_{i}^{(1)}}{A_{01}^{(1)} \varphi_{1}^{(1)}+\ldots+A_{0 n}^{(1)} \varphi_{n}^{(1)}+B_{0}^{(1)}}=\left\{\begin{array}{ccc}
\frac{1 . \varphi_{i}^{(1)}}{1 \cdot \varphi_{j}^{(1)}+a_{n+1}^{(1)}} & \text { for } & 1 \leq i<j \\
\frac{1}{1 . \varphi_{j}^{(1)}+a_{n+1}^{(1)}} & \text { for } & i=j \\
\frac{1 . \varphi_{i}^{(1)}+a_{i}^{(1)}}{1 . \varphi_{j}^{(1)}+a_{n+1}^{(1)}} & \text { for } & j<i \leq n
\end{array}\right.
$$

From (9) and (10), the assertion of theorem holds for $s=1$. Now, we assume that the assertion of the theorem holds by $s$, and we will show that the assertion holds for $s+1$. Note that $\kappa(s+1)$ is chosen by $\left(\varphi_{1}^{(s)}, \ldots, \varphi_{n}^{(s)}\right) \in \mathbb{L}_{\kappa(s+1)}^{(n)}$, 


$$
\begin{aligned}
& \frac{A_{i 1}^{(s+1)} \varphi_{1}^{(s+1)}+\ldots+A_{i n}^{(s+1)} \varphi_{n}^{(s+1)}+B_{i}^{(s+1)}}{A_{01}^{(s+1)} \varphi_{1}^{(s+1)}+\ldots+A_{0 n}^{(s+1)} \varphi_{n}^{(s+1)}+B_{0}^{(s+1)}} \\
& =\frac{\sum_{k=1}^{\kappa(s+1)} A_{i k}^{(s+1)} \frac{\varphi_{k}^{(s)}}{\varphi_{\kappa(s+1)}^{(s)}}+A_{i, \kappa(s+1)}^{(s+1)}\left(\frac{1}{\varphi_{\kappa(s+1)}^{(s)}}-a_{n+1}^{(s)}\right)+\sum_{k=\kappa(s+1)+1}^{n} A_{i k}^{(s+1)}\left(\frac{\varphi_{k}^{(s)}}{\varphi_{\kappa(s+1)}^{(s)}}-a_{n+1}^{(s)}\right)+B_{i}^{(s+1)}}{\sum_{k=1}^{\kappa(s+1)} A_{0 k}^{(s+1)} \frac{\varphi_{k}^{(s)}}{\varphi_{\kappa(s+1)}^{(s)}}+A_{0, \kappa(s+1)}^{(s+1)}\left(\frac{1}{\varphi_{\kappa(s+1)}^{(s)}}-a_{n+1}^{(s)}\right)+\sum_{k=\kappa(s+1)+1}^{n} A_{0 k}^{(s+1)}\left(\frac{\varphi_{k}^{(s)}}{\varphi_{\kappa(s+1)}^{(s)}}-a_{n+1}^{(s)}\right)+B_{0}^{(s+1)}} \\
& =\frac{\sum_{k=1}^{\kappa(s+1)} A_{i k}^{(s+1)} \frac{\varphi_{k}^{(s)}}{\varphi_{\kappa(s+1)}^{(s)}}+B_{i}^{(s)}\left(\frac{1}{\varphi_{\kappa(s+1)}^{(s)}}-a_{n+1}^{(s)}\right)+\sum_{k=\kappa(s+1)+1}^{n} A_{i k}^{(s)}\left(\frac{\varphi_{k}^{(s)}}{\varphi_{\kappa(s+1)}^{(s)}}-a_{n+1}^{(s)}\right)+B_{i}^{(s+1)}}{\sum_{k=1}^{\kappa(s+1)} A_{0 k}^{(s+1)} \frac{\varphi_{k}^{(s)}}{\varphi_{\kappa(s+1)}^{(s)}}+B_{0}^{(s)}\left(\frac{1}{\varphi_{\kappa(s+1)}^{(s)}}-a_{n+1}^{(s)}\right)+\sum_{k=\kappa(s+1)+1}^{n} A_{0 k}^{(s)}\left(\frac{\varphi_{k}^{(s)}}{\varphi_{\kappa(s+1)}^{(s)}}-a_{n+1}^{(s)}\right)+B_{0}^{(s+1)}}
\end{aligned}
$$

From (8),

$$
\begin{aligned}
& \frac{A_{i 1}^{(s+1)} \varphi_{1}^{(s+1)}+\ldots+A_{i n}^{(s+1)} \varphi_{n}^{(s+1)}+B_{i}^{(s+1)}}{A_{01}^{(s+1)} \varphi_{1}^{(s+1)}+\ldots+A_{0 n}^{(s+1)} \varphi_{n}^{(s+1)}+B_{0}^{(s+1)}} \\
& =\frac{\sum_{k=1}^{\kappa(s+1)} A_{i k}^{(s)} \frac{\varphi_{k}^{(s)}}{\varphi_{\kappa(s+1)}^{(s)}}+B_{i}^{(s)} \cdot \frac{1}{\varphi_{\kappa(s+1)}^{(s)}}+\sum_{k=\kappa(s+1)+1}^{n} A_{i k}^{(s)} \frac{\varphi_{k}^{(s)}}{\varphi_{\kappa(s+1)}^{(s)}}+A_{i, \kappa(s+1)}^{(s)}}{\sum_{k=1}^{n(s+1)} A_{0 k}^{(s)} \frac{\varphi_{k}^{(s)}}{\varphi_{\kappa(s+1)}^{(s)}}+B_{0}^{(s)} \cdot \frac{1}{\varphi_{\kappa(s+1)}^{(s)}}+\sum_{k=\kappa(s+1)+1}^{(s)} \frac{\varphi_{k}^{(s)}}{\varphi_{\kappa(s+1)}^{(s)}}+A_{0, \kappa(s+1)}^{(s)}} \\
& =\frac{A_{i 1}^{(s)} \varphi_{1}^{(s)}+\ldots+A_{i n}^{(s)} \varphi_{n}^{(s)}+B_{i}^{(s)}}{A_{01}^{(s)} \varphi_{1}^{(s)}+\ldots+A_{0 n}^{(s)} \varphi_{n}^{(s)}+B_{0}^{(s)}} \\
& =\varphi_{i} .
\end{aligned}
$$

Thus the assertion holds for $s+1$, completing the proof.

The vector $V_{0}^{(s)}=\left(\frac{B_{1}^{(s)}}{B_{0}^{(s)}}, \ldots, \frac{B_{n}^{(s)}}{B_{0}^{(s)}}\right)$ is called the $s$-th convergent of $\varphi=\left(\varphi_{1}, \ldots, \varphi_{n}\right)$ by the $\beta$-MJPA and $M^{(1)} \ldots M^{(s)}$ the matrices expansion by this algorithm.

Morover the expansion by the $\beta$-MJPA is said to be finite or infinite if $T_{\beta}^{s}\left(\varphi_{1}, \ldots, \varphi_{n}\right)=$ $(0, \ldots, 0)$ for some $s \geq 0$ or $T_{\beta}^{s}\left(\varphi_{1}, \ldots, \varphi_{n}\right) \neq(0, \ldots, 0)$ for any $s \geq 0$, respectively. 


\section{Convergence of $A$-Modified Jacobi-Perron algorithm over the field of formal power series}

Now, we give the main result.

Theorem 2. Let $\varphi=\left(\varphi_{1}, \ldots, \varphi_{n}\right) \in \mathbb{L}^{n}$ and $V_{0}^{(s)}=V_{0}^{(s)}(\varphi)$ for all $s \geq 1$, then the sequence $\left(V_{0}^{(s)}\right)_{s \geq 1}$ converges to $\varphi$.

In order to prove this theorem we need the following lemma

Lemma 2. For any sequence $M^{(1)}, \cdots, M^{(s+1)}, \cdots$ of the form (5)

$$
\left|B_{0}^{(s)}\right|\left|\frac{B_{i}^{(s+1)}}{B_{0}^{(s+1)}}-\frac{B_{i}^{(s)}}{B_{0}^{(s)}}\right| \leq e^{-1}
$$

holds for any $s \geq 1$.

Proof. We prove this result by using the mathematical induction on $s$. Note that $\kappa(s)=\min _{1 \leq i \leq n+1}\left\{i: m_{i, n+1}^{(s)} \neq 0\right\}$ where $m_{i, n+1}^{(s)}$ is the $(i, n+1)$ component of $M^{(s)}$. Then if $1 \leq \kappa(1)<\kappa(2)$,

$$
\left|\frac{B_{i}^{(2)}}{B_{0}^{(2)}}-\frac{B_{i}^{(1)}}{B_{0}^{(1)}}\right|=\left|\frac{a_{i}^{(2)}}{a_{n+1}^{(1)} a_{n+1}^{(2)}}\right| \text { for } 1 \leq i \leq n .
$$

Since $\operatorname{deg} a_{n+1}^{(s)} \geq 1$ and $\operatorname{deg} a_{n+1}^{(s)} \geq \operatorname{deg} a_{i}^{(s)}, 1 \leq i \leq n$, for $s \geq 1$, we have

$$
\left|B_{0}^{(1)}\right|\left|\frac{B_{i}^{(2)}}{B_{0}^{(2)}}-\frac{B_{i}^{(1)}}{B_{0}^{(1)}}\right| \leq e^{-1}
$$

Moreover, if $\kappa(1)=\kappa(2)$, we have

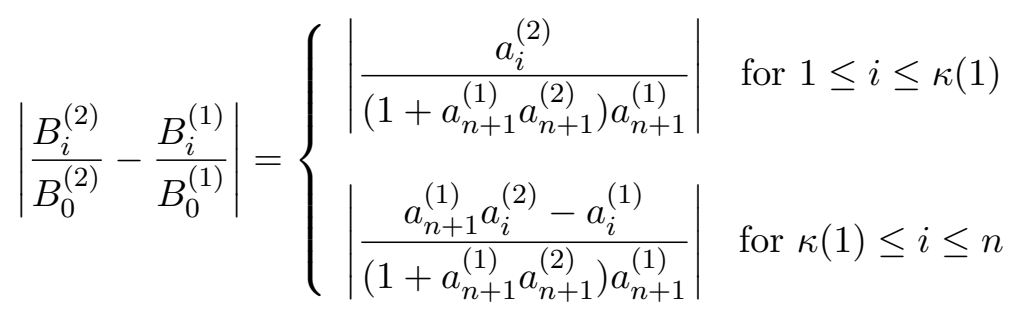

and if $\kappa(2)<\kappa(1) \leq n$, we have

$$
\left|\frac{B_{i}^{(2)}}{B_{0}^{(2)}}-\frac{B_{i}^{(1)}}{B_{0}^{(1)}}\right|= \begin{cases}\left|\frac{a_{i}^{(2)}}{\left(a_{n+1}^{(1)} a_{n+1}^{(2)}+a_{\kappa(1)}^{(2)}\right.}\right| & \text { for } 1 \leq i \leq \kappa(1) \\ \left|\frac{a_{n+1}^{(1)} a_{i}^{(2)}-a_{i}^{(1)} a_{\kappa(1)}^{(2)}}{\left(a_{\kappa(1)}^{(2)}+a_{n+1}^{(1)} a_{n+1}^{(2)}\right) a_{n+1}^{(1)}}\right| & \text { for } \kappa(1) \leq i \leq n\end{cases}
$$


Then similarly, we have

$$
\left|B_{0}^{(1)}\right|\left|\frac{B_{i}^{(2)}}{B_{0}^{(2)}}-\frac{B_{i}^{(1)}}{B_{0}^{(1)}}\right| \leq e^{-1}
$$

Now we suppose the assertion of (Lemma 2) holds by $s-1$. For $s \geq 2$

$$
\begin{aligned}
\left|\frac{B_{i}^{(s+1)}}{B_{0}^{(s+1)}}-\frac{B_{i}^{(s)}}{B_{0}^{(s)}}\right| & =\left|\frac{A_{i \kappa(s+1)}^{(s)}+\sum_{k=\kappa(s+1)+1}^{n} a_{k}^{(s+1)} A_{i k}^{(s)}+a_{n+1}^{(s+1)} B_{i}^{(s)}}{A_{0 \kappa(s+1)}^{(s)}+\sum_{k=\kappa(s+1)+1}^{n} a_{k}^{(s+1)} A_{0 k}^{(s)}+a_{n+1}^{(s+1)} B_{0}^{(s)}}-\frac{B_{i}^{(s)}}{B_{0}^{(s)}}\right| \\
& =\left|\frac{A_{i \kappa(s+1)}^{(s)} B_{0}^{(s)}-A_{0 \kappa(s+1)}^{(s)} B_{i}^{(s)}+\sum_{k=\kappa(s+1)+1}^{n} a_{k}^{(s+1)}\left(A_{i k}^{(s)} B_{0}^{(s)}-A_{0 k}^{(s)} B_{i}^{(s)}\right)}{\left(A_{0 \kappa(s+1)}^{(s)}+\sum_{k=\kappa(s+1)+1}^{n} a_{k}^{(s+1)} A_{0 k}^{(s)}+a_{n+1}^{(s+1)} B_{0}^{(s)}\right) B_{0}^{(s)}}\right|
\end{aligned}
$$

Using the fact that $\operatorname{deg} a_{k}^{(s+1)} A_{0 k}^{(s)}<a_{n+1}^{(s)} B_{0}^{(s)}$

$$
\begin{aligned}
\left|\frac{B_{i}^{(s+1)}}{B_{0}^{(s+1)}}-\frac{B_{i}^{(s)}}{B_{0}^{(s)}}\right| & =\frac{\left|\sum_{k=\kappa(s+1)}^{n} a_{k}^{(s+1)}\left(A_{i k}^{(s)} B_{0}^{(s)}-A_{0 k}^{(s)} B_{i}^{(s)}\right)\right|}{\left|a_{n+1}^{(s+1)}\left(B_{0}^{(s)}\right)^{2}\right|} \\
& =\frac{1}{\left|a_{n+1}^{(s+1)} B_{0}^{(s)}\right|}\left|\sum_{k=\kappa(s+1)}^{n} a_{k}^{(s+1)} A_{0 k}^{(s)}\left(\frac{A_{i k}^{(s)}}{A_{0 k}^{(s)}}-\frac{B_{i}^{(s)}}{B_{0}^{(s)}}\right)\right|
\end{aligned}
$$

By (6) and (7), we replace $A_{i k}^{(s)}$ by $B_{i}^{\left(l_{k}\right)}$, for some $l_{k}, l_{k}<s$.

$$
\begin{aligned}
\left|B_{0}^{(s)}\right|\left|\frac{B_{i}^{(s+1)}}{B_{0}^{(s+1)}}-\frac{B_{i}^{(s)}}{B_{0}^{(s)}}\right| & =\frac{1}{\left|a_{n+1}^{(s+1)}\right|}\left|\sum_{k=\kappa(s+1)}^{n} a_{k}^{(s+1)} B_{0}^{\left(l_{k}\right)}\left(\frac{B_{i}^{\left(l_{k}\right)}}{B_{0}^{\left(l_{k}\right)}}-\frac{B_{i}^{(s)}}{B_{0}^{(s)}}\right)\right| \\
& \leq \frac{1}{\left|a_{n+1}^{(s+1)}\right|}\left|\sum_{k=\kappa(s+1)}^{n} a_{k}^{(s+1)} B_{0}^{\left(l_{k}\right)} \sum_{k=1}^{s-1}\left(\frac{B_{i}^{(l)}}{B_{0}^{(l)}}-\frac{B_{i}^{(l+1)}}{B_{0}^{(l+1)}}\right)\right| \\
& \leq \frac{1}{\left|a_{n+1}^{(s+1)}\right|} \max _{1 \leq k \leq s-1} \max _{k \leq l \leq s-1}\left|B_{0}^{(k)}\right|\left|\frac{B_{i}^{(l)}}{B_{0}^{(l)}}-\frac{B_{i}^{(l+1)}}{B_{0}^{(l+1)}}\right| \\
& \leq e^{-2} \leq e^{-1}
\end{aligned}
$$


Then, from the assumption of the induction,

$$
\left|B_{0}^{(s)}\right|\left|\frac{B_{i}^{(s+1)}}{B_{0}^{(s+1)}}-\frac{B_{i}^{(s)}}{B_{0}^{(s)}}\right| \leq e^{-1}
$$

completing the proof.

Proof. (of theorem 2). We see

$$
\begin{aligned}
\left|\varphi_{i}-\frac{B_{i}^{(s)}}{B_{0}^{(s)}}\right| & =\left|\frac{A_{i 1}^{(s)} \varphi_{1}^{(s)}+\ldots+A_{i n}^{(s)} \varphi_{n}^{(s)}+B_{i}^{(s)}}{A_{01}^{(s)} \varphi_{1}^{(s)}+\ldots+A_{0 n}^{(s)} \varphi_{n}^{(s)}+B_{0}^{(s)}}-\frac{B_{i}^{(s)}}{B_{0}^{(s)}}\right| \\
& =\left|\frac{\sum_{k=1}^{n}\left(A_{i k}^{(s)} B_{0}^{(s)}-A_{0 k}^{(s)} B_{i}^{(s)}\right) \varphi_{k}^{(s)}}{\left(A_{01}^{(s)} \varphi_{1}^{(s)}+\ldots+A_{0 n}^{(s)} \varphi_{n}^{(s)}+B_{0}^{(s)}\right) B_{0}^{(s)}}\right| \\
& =\left|\frac{\sum_{k=1}^{n}\left(\frac{A_{i k}^{(s)}}{A_{0 k}^{(s)}}-\frac{B_{i}^{(s)}}{B_{0}^{(s)}}\right) A_{0 k}^{(s)} \varphi_{k}^{(s)}+\ldots+A_{0 n}^{(s)} \varphi_{n}^{(s)}+B_{0}^{(s)}}{A_{01}^{(s)}}\right|
\end{aligned}
$$

For each $k, 1 \leq k \leq n$, there exists an increasing sequence $l_{k}, l_{k}<s$, such that $j\left(l_{k}\right)=k$, one gets $A_{i k}^{(s)}=B_{i}^{\left(l_{k}\right)}$.

Then we have 


$$
\begin{aligned}
\left|\varphi_{i}-\frac{B_{i}^{(s)}}{B_{0}^{(s)}}\right| & =\left|\frac{\sum_{k=1}^{n}\left(\frac{A_{i k}^{(s)}}{A_{0 k}^{(s)}}-\frac{B_{i}^{(s)}}{B_{0}^{(s)}}\right) A_{0 k}^{(s)} \varphi_{k}^{(s)}}{A_{01}^{(s)} \varphi_{1}^{(s)}+\ldots+A_{0 n}^{(s)} \varphi_{n}^{(s)}+B_{0}^{(s)}}\right| \\
& =\left|\frac{\sum_{k=1}^{n}\left(\frac{A_{i k}^{\left(l_{k}\right)}}{A_{0 k}^{\left(l_{k}\right)}}-\frac{B_{i}^{(s)}}{B_{0}^{(s)}}\right) B_{0}^{\left(l_{k}\right)} \varphi_{k}^{(s)} \mid}{A_{01}^{(s)} \varphi_{1}^{(s)}+\ldots+A_{0 n}^{(s)} \varphi_{n}^{(s)}+B_{0}^{(s)}}\right| \\
& =\frac{\sum_{k=1}^{n}\left|B_{0}^{\left(l_{k}\right)}\right|\left|\left(\frac{A_{i k}^{\left(l_{k}\right)}}{A_{0 k}^{\left(l_{k}\right)}}-\frac{B_{i}^{(s)}}{B_{0}^{(s)}}\right) \varphi_{k}^{(s)}\right|}{\left|A_{01}^{(s)} \varphi_{1}^{(s)}+\ldots+A_{0 n}^{(s)} \varphi_{n}^{(s)}+B_{0}^{(s)}\right|} \\
& \leq \frac{\max _{1 \leq l \leq s-1}\left|B_{0}^{\left(l_{k}\right)}\right|\left|\left(\frac{A_{i k}^{\left(l_{k}\right)}}{A_{0 k}^{\left(l_{k}\right)}}-\frac{B_{i}^{(s)}}{B_{0}^{(s)}}\right) \varphi_{k}^{(s)}\right|}{\left|A_{01}^{(s)} \varphi_{1}^{(s)}+\ldots+A_{0 n}^{(s)} \varphi_{n}^{(s)}+B_{0}^{(s)}\right|}
\end{aligned}
$$

Using (Lemma 2), we get

$$
\left|\varphi_{i}-\frac{B_{i}^{(s)}}{B_{0}^{(s)}}\right| \leq e^{-1} \frac{\left|\varphi_{k}^{(s)}\right|}{\left|A_{01}^{(s)} \varphi_{1}^{(s)}+\ldots+A_{0 n}^{(s)} \varphi_{n}^{(s)}+B_{0}^{(s)}\right|}
$$

Since $\operatorname{deg} B_{0}^{(s)}=\sum_{k=1}^{s} \operatorname{deg} a_{n+1}^{(k)} \geq s$, then, for any $\epsilon>0$, there exists $s_{0} \geq 1$ such that

$$
\left|\varphi_{i}-\frac{B_{i}^{(s)}}{B_{0}^{(s)}}\right| \leq \frac{e^{-1}}{\left|B_{0}^{(s)}\right|}<\varepsilon, \text { for any } s \geq s_{0}
$$

This implies

$$
\lim _{s \longrightarrow \infty} \frac{B_{i}^{(s)}}{B_{0}^{(s)}}=\varphi_{i} \text { for } 1 \leq i \leq n
$$

which proves the theorem. 


\section{References}

[1] A. Chandoul. Simpler proof of convergence of Brun algorithm over the field of formal power series, International Journal of Algebra, v. 5, p. 25-30, 2011.

[2] A. Chandoul. On continued fractions over the field of formal power series, International Journal of Contomporary Mathematical Sciences, v. 6, p. 1351-1356, 2011.

[3] A. Chandoul, On periodic Jacobi-Perron algorithm over the field of formal power series, New Trends in Mathematical Science, v. 1, p. 145-152, 2018.

[4] H. Ben amar and A. Chandoul, Convergence of the Brun algorithm over the field of formal power series, Journal of number theory 197, 621-631, 2009.

[5] A. Chandoul et al. On periodic P-continued fraction having period lenght one, Bulletin of the Korean Mathematical Society, v. 50, p. 1623-1630, 2013.

[6] A. Chandoul et al. On the Continued Fraction Expansion of Fixed Period in Finite Fields, Canadian Mathematical Bulletin, v. 58, p. 704-712, 2015.

[7] M. Jellali et al. Beta-continued fraction over Laurent series, Pub Math. Debrecen 77/3-4,443-463, 2010.

[8] E. Artin, Quadratische Körper im Gebiete der höhern Kongruenzen I., Arithmetischer Teil. II. (Analytischer Teil.), 1924.

[9] V. Berthé and H. Nakada. On continued fraction expansions in positive characteristic : Equivalence relations and some metric properties, Expo. Math., 18(4), 257-284, 2000 .

[10] M. Fochs. An analogue of a theorem of Szusz for formal Laurent series over finite fields, Journal of Number Theory, 101(1), 105-130, 2003.

[11] K. Schmidt. On periodic expansions of Pisot numbers and Salem numbers. Bull. London Math. Soc., 12(4):269-278, 1980. 